\title{
Asia Pacific Stroke Conference 2021: Stroke Care in Challenging Times
}

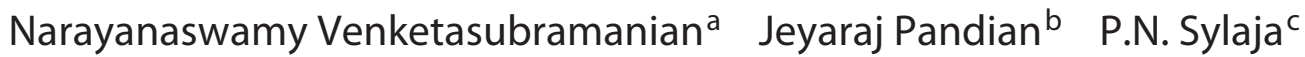

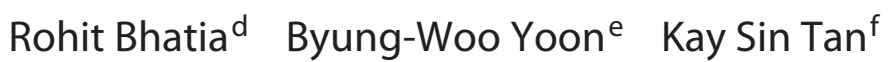 \\ aRaffles Neuroscience Centre, Raffles Hospital, Singapore, Singapore; bepartment of Neurology, Christian \\ Medical College, Ludhiana, India; 'Department of Neurology, Sree Chitra Tirunal Institute for Medical Sciences and \\ Technology, Trivandrum, India; dDepartment of Neurology, All India Institute of Medical Sciences, New Delhi, India; \\ eDepartment of Neurology, Uijeongbu Eulji Medical Center, Eulji University, Uijeongbu, Republic of Korea; ${ }^{\text {fDivision }}$ \\ of Neurology, Department of Medicine, Faculty of Medicine, University of Malaya, Kuala Lumpur, Malaysia
}

The flagship Continuing Medical Education (CME) event for the Asia Pacific Stroke Organization (APSO) has been the annual Asia Pacific Stoke Conference (APSC) since 2011. It has been held in various cities throughout the Asia-Pacific region. The broad-ranged programs comprise workshops, plenary sessions, focused symposia, satellite sessions, debates, free paper platform and poster sessions, educational courses, and sponsored symposia, provided by a geographically diverse expert faculty. APSC 2021 is being held in Chennai, India, from 9 to 11 December 2021, using an online format [1]. It will be organized by the Indian Stroke Association, with the most appropriate theme "Stroke Care in Challenging Times." The registration fee has been kept low, encouraging a strong attendance. As has been done for past APSCs, APSO awards will be given to the 10 best abstracts submitted from lowand low-middle-income countries.

The sessions in APSC 2021 cover a broad and clinically relevant topics including epidemiology, primary and secondary prevention, imaging, neurosonology, telestroke, vascular cognitive impairment, acute stroke

karger@karger.com

(c) 2021 S. Karger AG, Basel

www.karger.com/ced

Karger management, paediatric stroke, rehabilitation, and the current hot topic of COVID-19 and stroke. Symposia have also been organized on issues peculiar to the AsiaPacific region including intracerebral haemorrhage, intracranial atherosclerosis, infections, moya-moya disease, cerebral venous thrombosis, radiation-induced vasculopathy, alternative and complementary therapy, and challenges to health-care systems. A new addition is a symposium devoted solely to stroke support groups.

The many high-quality scientific abstracts range from case reports to clinical trials to meta-analysis. These are presented here in this Supplement, also accessible on the APSO website, as have been the abstracts of all past APSCs. Of the 154 submitted abstracts, the largest number came from India (69.5\%) and Indonesia (14.3\%) (shown in Fig. 1). Of interest are articles on stroke and stroke care during COVID-19, cerebral venous thrombosis with and without COVID-19, stroke awareness, income status and stroke burden, clinical scores to diagnose large artery occlusion, CT and CT-perfusion, Alberta stroke programme early CT score (ASPECTS) MRI, ultrasound 
Fig. 1. APSC 2021 - number of abstracts submitted by country. APSC, Asia Pacific Stroke Conference.

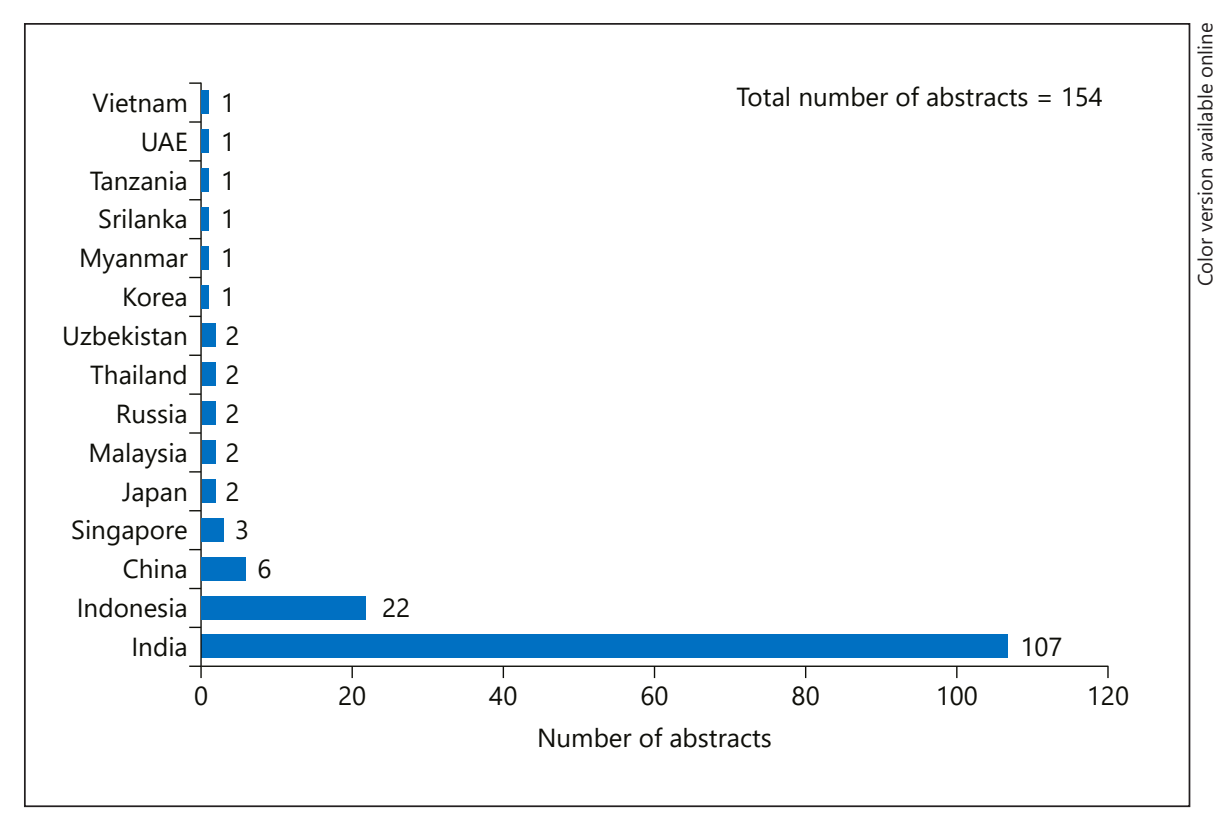

probes, TCD pulsatility index, use of tenecteplase in India, thrombectomy, anticoagulation in patients with cerebral microbleeds, delayed versus early use of NOAC, stem cell treatment, robotics, transcranial direct current stimulation for stroke recovery and challenges in postdischarge care.

These abstracts reflect the varied and high-quality research in the Asia-Pacific region, which we hope will translate to full publications, and with the sharing of new knowledge, to better patient care and outcomes. Many of these topics serve to educate and instruct with ideas as well as reminders for all levels of stroke practitioners. This meeting is also relevant for researchers to gain insight into geographically specific issues and research gaps.

In a broader context, stroke is a major cause of death and disability globally, even more so in the Asia Pacific region $[2,3]$. The current COVID-19 pandemic has added even more stress to health-care systems in large and small countries already burdened by issues including accessibility and cost [4]. Health-care planning will need a holistic approach with adequate future-proofing for future challenges to health-care provision, including providing health-care professionals (HCPs) with the required expertise, the infrastructure, and the numbers to optimally manage their stroke patients and to adequately support their families and care givers.

The main thrust of APSO has been in contributing to CME of HCPs [5, 6]. APSO has provided education grants to member societies to hold local stroke conferences, funded APSO visiting professorships, endorsed nationallevel stroke conferences, enhanced the APSO website with links to webpages of major stroke journals such as Cerebrovascular Diseases, stroke support organizations, clinical practice guidelines, and most recently held webinars on clinically relevant issues.

APSC 2022 will be held in Taipei, Taiwan, from 25 to 27 November 2022. We welcome all to attend for another exciting and fullfilling CME event. APSO will open future bids for the hosting of Asia Pacific Stroke Conference for 2023 and beyond, and will welcome expression of interest from members.

\section{Acknowledgements}

The authors would like to thank all the member countries of the APSO, the members of the organizing committee and scientific committee for the 2021 edition of the APSC, as well as all the speakers, co-chairpersons, participants, and sponsors for their support in this meeting during these unprecedented times.

\section{Conflict of Interest Statement}

The authors have no conflicts of interest to declare.
IV

Cerebrovasc Dis 2021;50(suppl 1):III-V DOI: $10.1159 / 000520615$
Venketasubramanian/Pandian/Sylaja/ Bhatia/Yoon/Tan 


\section{Funding Sources}

Thie work performed for this paper did not require funding.

\section{Author Contributions}

N.V. conceptualized the paper, wrote the paper, and approved the final version. J.P., P.N.S., R.B., and B.W.Y. provided critical review and approved the final version. K.S.T. conceptualized the paper, provided critical review, and approved the final version.

\section{References}

1 Asia Pacific Stroke Conference 2021. Available from: www.apscindia.com.

2 Venketasubramanian N, Yoon BW, Pandian J, Navarro JC. Stroke epidemiology in South, East, and South-East Asia: a review. J Stroke. 2017 Sep;19(3):286-94.
3 Venketasubramanian N. Stroke epidemiology in Oceania: a review. Neuroepidemiology. 2021 Feb 18:1-10.

4 Sylaja PN, Srivastava MVP, Shah S, Bhatia R, Khurana D, Sharma A, et al. The SARSCoV-2/COVID-19 pandemic and challenges in stroke care in India. Ann N Y Acad Sci. 2020 Aug; 1473(1):3-10.
5 Tan KS, Lin RT, Yoon BW, Suwanwela N, Mehndiratta MM, Venketasubramanian N. Organizational update: Asia Pacific stroke organization (APSO). Stroke. 2021.

6 Asia Pacific Stroke Organisation. Education. Available from: https://www.theapso.com/ education. 